\title{
Low-Energy Ions from Laser-Cooled Atoms
}

\author{
G. Shayeganrad, ${ }^{1, *}$ A. Fioretti, ${ }^{2,3}$ I. Guerri, ${ }^{1}$ F. Tantussi, ${ }^{1,2,3,}$ D. Ciampini, ${ }^{1,2,3}$ M. Allegrini,,${ }^{1,2,3}$ M. Viteau, ${ }^{4}$ and F. Fuso ${ }^{1,2,3}$ \\ ${ }^{1}$ Dipartimento di Fisica Enrico Fermi, Università di Pisa, 56127 Pisa, Italy \\ ${ }^{2}$ Istituto Nazionale di Ottica, INO-CNR, 56124 Pisa, Italy \\ ${ }^{3}$ Consorzio Nazionale Interuniversitario per le Scienze Fisiche della Materia, \\ CNISM, Sezione di Pisa, 56127 Pisa, Italy \\ ${ }^{4}$ Orsay Physics, TESCAN Orsay, 13710 Fuveau, France
}

(Received 12 June 2015; revised manuscript received 19 April 2016; published 27 May 2016)

\begin{abstract}
We report the features of an ion source based on two-color photoionization of a laser-cooled cesium beam outsourced from a pyramidal magneto-optical trap. The ion source operates in continuous or pulsed mode. At acceleration voltages below $300 \mathrm{~V}$, it delivers some ten ions per bunch with a relative energy spread $\Delta U_{\mathrm{rms}} / U \simeq 0.032$, as measured through the retarding field-energy-analyzer approach. Spacecharge effects are negligible thanks to the low ion density attained in the interaction volume. The performances of the ion beam in a configuration using focused laser beams are extrapolated on the basis of the experimental results. Calculations demonstrate that our low-energy and low-current ion beam can be attractive for the development of emerging technologies requiring the delivery of a small amount of charge, down to the single-ion level and its eventual focusing in the $10-\mathrm{nm}$ range.
\end{abstract}

DOI: $10.1103 /$ PhysRevApplied.5.054020

\section{INTRODUCTION}

Monochromatic ion beams are invaluable tools in material science in the emerging nanotechnology industry and in studies of biological materials. In these domains, where ions are used to modify, image, or analyze surfaces and materials, the ability to convey large ion currents into smaller and smaller spot sizes is considered as a primary figure of merit [1]. State-of-the-art focused ion beams (FIBs) are commercially available, based mainly on plasma, liquid-metal tip, or helium-ion sources for very large, intermediate, and relatively low currents, respectively [2].

Alongside those well-established techniques (a recent review on FIB methods and applications can be found in Ref. [3]), a number of applications are presently growing, requiring, in turn, tailored ion sources. For instance, on the other extreme of the current range, single-ion delivery and implantation onto a surface with nanometric precision opens exciting research possibilities and leads to the ultimate frontiers of the solitary-dopant optoelectronicssolotronics-for engineering few-atom devices [4-6]. In this endeavor, one of the major goals is the realization of a solid-state quantum processor based on single-implantedqubit carriers like color centers in diamond or phosphorous dopants in silicon. For this process, the figure of merit depends on parameters such as the feasibility, resolution, speed, and scalability of the implantation. Remarkable results have been attained by combining different

\footnotetext{
*Present address: Fakultät Physik, Technische Universität Dortmund, 44227 Dortmund, Germany.

${ }^{\dagger}$ Present address: Istituto Italiano di Tecnologia, IIT, 16136 Genova, Italy.
}

techniques such as scanning tunneling microscopy and atomic force microscopy with ion guns or FIBs in order to control the process and have a direct imaging of the modified surface soon after implantation [7-9]. Lowcurrent beams attract interest also for advanced biophysical applications. Besides the traditional role of implantation in radiobiology [10], single-ion implantation into living cells has been proposed as a tool for viability analyses aimed at designing functional cellular modifications [11].

Another key issue in ion-beam technology deals with the use of small acceleration voltages compared to the typical kilo-electron-volt or tens of kilo-electron-volt FIB energies. Limiting the kinetic energy considerably reduces the structural damage of the target. Decreasing the kinetic energy is a must for doping thin and brittle material layers including, for instance, carbon nanotubes and few-layered graphene [12-14]. A similar requirement holds also for techniques making use of heavy ion beams as secondaryion mass spectrometry and reactive-ion-scattering spectroscopy (RIS) $[15,16]$. Here, reduction of the atomic mixing region in depth, surface penetration, and efficient disruption requires ions at sub-kilo-electron-volt kinetic energy, leading to greater depth resolution [17,18]. RIS using low-energy $(U<100 \mathrm{eV}) \mathrm{Cs}^{+}$ions demonstrates its potential for detailed surface molecular analysis. Furthermore, low-current ion beams with a kinetic energy of the same order of magnitude or slightly larger than the chemical bond energy can pave the way for further developments in technologies based on ion reactions with soft matter [19].

Conventional instrumentation can be adapted to such emerging applications. However, sophisticated modifications 
$[7,12]$ and a careful design of the ion optics setup [1] to avoid degradation of the chromatic properties at low-energy operation are required. Furthermore, conventional ion beams suffer frequently from space-charge and related effects, which can hamper the attainment of arbitrarily small focal spots. Since space charge stems from the large ion density in the initial stage of beam creation, reducing the delivered current through, for instance, skimming by nanosized apertures [7] or blanking by pulsed electric fields [4], cannot improve the beam performance. Thus, the development of compact sources unaffected by space charge able to deliver a limited and well-controlled amount of ions and to operate at low-acceleration voltage represents a challenging task worth exploring. In view of technological applications, simplified and robust configurations enabling a straightforward integration with the components needed for focusing and guiding the ion beam are obviously preferred.

In this paper, we demonstrate the feasibility of a lowenergy and low-current ion source based on the photoionization of a laser-cooled cesium atom beam outsourced from a pyramidal magneto-optical trap (MOT).

Ion beams with low emittance, high brightness, and wellcontrolled energy have been produced from laser-cooled and trapped atoms [20-27] aimed at replacing conventional ion sources for typical FIB applications, i.e., surface modifications and ion imaging. A recent review on bright focused-ion-beam sources based on laser-cooling technologies can be found in Ref. [28]. The main advantages provided by laser-cooled atoms are (i) a very low initial temperature satisfying the need for low emittance and high monochromaticity required to attain nanometer focusing of the extracted ions, (ii) an effective manipulation and control of the atoms by laser, rf, or static fields, allowing quasideterministic and on-demand delivery of single ions $[29,30]$ through approaches in common with ion manipulation [31], and (iii) a variety of atomic species have been successfully laser cooled enabling a wealth of possible ion beams that can tailor a specific need or application.

Recently, the performance of an ion source based on a laser-cooled and compressed atom beam outsourced from a Knudsen (effusive) cell has been predicted [32], suggesting the attainment of impressive improvements with respect to the present technologies [33]. The capabilities of low-energy ion beams in terms of spatial resolution and surface-sensitive compositional analysis have been demonstrated in a lithium-ion microscope operated at relatively low $(500 \mathrm{~V}-5 \mathrm{kV})$ acceleration voltage [34].

While sharing similar methods and concepts, our approach is not yet an alternative route to ion-source replacement in conventional FIBs. Indeed, in the present configuration, the stringent requirements in the beam brightness and ion current for the operation of a FIB are not met. Here, we fully exploit the distinctive ability of the pyramidal MOT in producing a stable flow of atoms with a small particle density and controlled properties, including low divergence and reduced longitudinal velocity. We then demonstrate a low-energy and low-current ion source virtually unaffected by space-charge effects that is able to operate in continuous and pulsed modes. Further developments of this ion beam are also discussed in view of possible applications.

\section{EXPERIMENTAL SETUP}

The core of our setup is a pyramidal MOT. Compared to conventional 2D MOTs, its design is inherently much more suitable for technological applications [35,36], as it uses a single trapping and cooling laser beam. This suitability is demonstrated also by its role in the development of integrated atom clocks and atom-chip devices $[37,38]$. The pyramidal MOT enables a simplified optical setup, strongly reducing the amount of optical components and the care for their alignment. This reduction, in turn, improves the stability and the resilience of the system. Furthermore, the pyramid assembly can be easily integrated into the components for ion guiding and focusing, typically hosted in cylindrical-symmetric standard ultra-high-vacuum (UHV) chambers and can be scaled in size in order to fit with specific experimental requirements.

Details of the laser-cooled atom-beam production method and the relevant experimental setup can be found in Ref. [39]. Briefly, a Cs beam leaves a pyramidal MOT and propagates horizontally in a vacuum chamber with a residual pressure of approximately $10^{-9} \mathrm{mbar}$. The pyramidal MOT [40] consists of a specific assembly of mirrors and prisms arranged to form a hollow reflective pyramid (3.7cm edge size) hosted in a CF-100 UHV stainless-steel chamber with a single window for optical access. Laser cooling and trapping of the background atoms is achieved by shining a single large-size beam of laser radiation (consisting of superposed trapping and repumping radiations) onto the inner surface of the pyramid in the presence of the nonhomogeneous static magnetic field produced by a pair of coils in the anti-Helmoltz configuration. An apical hole with a $1 \times 2 \mathrm{~mm}^{2}$ surface allows laser-manipulated atoms to be continuously released from the pyramidal MOT. In typical operating conditions, the flux of cold atoms is approximately $5 \times 10^{8}$ atoms/s, ultimately limited by the low-Cs background pressure within the pyramid volume and by the available trapping laser power. In our setup, background atoms are produced by electrically operated Cs dispensers (SAES Getters) fed with a 4.4-A current. The atom beam has an average longitudinal velocity $v_{z}$ in the $10-25 \mathrm{~m} / \mathrm{s}$ range depending on the operating parameters $\left(v_{z} \approx 23 \mathrm{~m} / \mathrm{s}\right.$ for the present experiment), a longitudinal velocity spread on the order of a few meters per second, and a divergence of approximately $30 \mathrm{mrad}$. Such a relatively large divergence, typical, to the best of our knowledge, for all atom beam sources based on magneto-optical trapping (see, e.g., Ref. [25]), requires a further collimation stage. As shown in Fig. 1(a), at the 


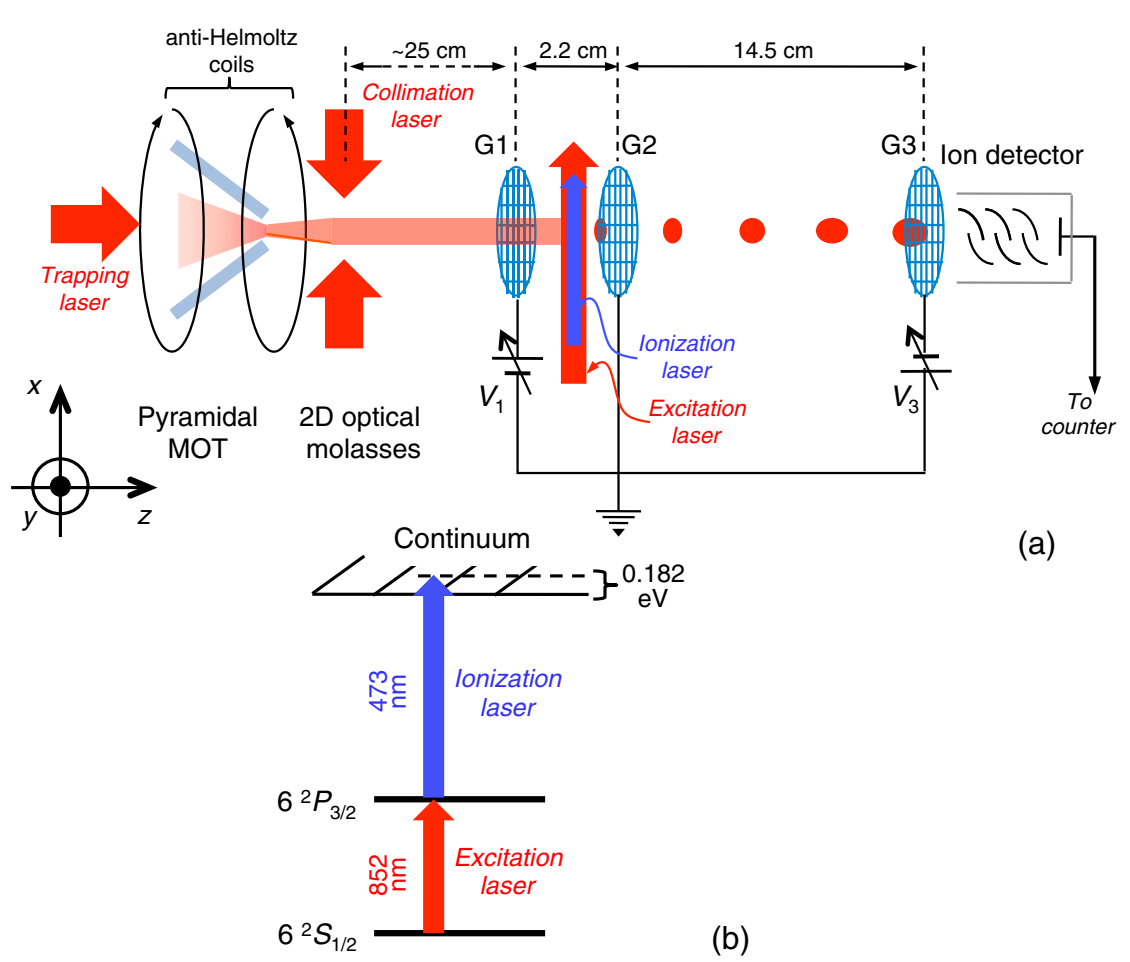

FIG. 1. Sketch of the experimental setup (a) and simplified Cs-energy-level diagram with indicated excitation and ionization paths (b). In the drawing of panel (a), not to scale, G1, G2, and G3 are metal grids. Production of ions occurs in the region between G1 and G2 thanks to interaction with the excitation and ionization laser beams. The circular and elliptical dots are a pictorial representation of the ion-bunch evolution.

pyramid exit, the atom beam is transversally cooled by a $2 \mathrm{D}$ optical molasses reducing its transverse velocity to $v_{\perp} \approx 9 \mathrm{~cm} / \mathrm{s}$, which corresponds to a residual divergence $\alpha \approx 4 \mathrm{mrad}$ [39]. This divergence is equivalent to a transverse kinetic temperature of the atoms $T_{\perp} \sim 10^{-4} \mathrm{~K}$, similar to the Cs Doppler cooling temperature [41].

Another key feature of our setup is the simple photoionization scheme making use of low-cost, low-power, compact, solid-state lasers. As shown in Fig. 1(a), at approximately $25 \mathrm{~cm}$ downstream from the collimation zone, the atom beam enters a region where it interacts with the excitation and ionization laser beams, which are directed into the chamber perpendicular to the atoms and can be superposed either perpendicular or parallel to each other. Unless otherwise stated, the copropagating laser configuration is used.

Ionization of ground-state atoms occurs in a two-color process, as sketched in the simplified energy-level diagram of Fig. 1(b). Atomic excitation is performed using an external-cavity diode laser injecting a slave laser tuned on the $6{ }^{2} S_{1 / 2}(F=4) \rightarrow 6{ }^{2} P_{3 / 2}\left(F^{\prime}=5\right)$ hyperfine (HF) transition. The chosen transition has the largest line strength $(A=11 / 27)$ and hyperfine branching coefficient $(\Pi=1)$ among the transitions of the D2 line of cesium [41]. Frequency stability of the excitation laser is maintained by locking it to the atomic line through the saturatedabsorption-spectroscopy technique. Ionization is obtained with a frequency-doubled diode-pumped Nd:YAG laser at $473 \mathrm{~nm}$ with a maximum power around $30 \mathrm{~mW}$ (MBL-473). The laser power is changed by a variable neutral density filter and measured by a calibrated power meter, while the laser spot widths are measured by a CCD beam profiler
(DataRay WinCamD). The ionization and excitation laser beams show a Gaussian profile with $\mathrm{rms}$ radii $\sigma_{\text {rms,ion }}=$ $(605 \pm 15) \mu \mathrm{m}$ and $\sigma_{\text {rms,exc }}=(700 \pm 25) \mu \mathrm{m}$, respectively. The atom density in the interaction region determined by absorption spectroscopy is typically $n \approx 5 \times 10^{5} \mathrm{~cm}^{-3}$, and the effective transverse width of the atom beam measured by means of fluorescence imaging is $\sigma_{\text {rms,beam }} \approx 3.4 \mathrm{~mm}$. In our experiment, no focusing is used for both laser beams, enabling a large signal-to-noise ratio in the measurements. The interaction volume can be roughly approximated with a cylinder having height corresponding to the transverse width of the atom beam and base surface defined by the width of the ionization laser.

In the interaction volume, atoms are promoted to the 6 ${ }^{2} P_{3 / 2}$ level by the excitation laser and then photoionized by the 2.6-eV energy of photons at $473 \mathrm{~nm}$ [see Fig. 1(b)]. This results in an excess energy of $0.182 \mathrm{eV}$ above threshold, which is taken almost completely by the electrons, owing to their small mass compared to that of the ions. A calculation based on momentum and energy conservation indicates that, consequent to ionization, ions get an extra energy $U_{\mathrm{exc}} \approx 0.7 \mu \mathrm{eV}$ corresponding to a velocity $v_{\mathrm{exc}} \simeq 1 \mathrm{~m} / \mathrm{s}$. Such a value is intermediate between the atom velocity along the transverse and longitudinal directions. However, in our experiment, ions are extracted from the interaction region at a minimum energy on the order of a few electron volts. Therefore, the contribution of $U_{\mathrm{exc}}$ is negligible in ruling the energy of the extracted ions, even if it plays a relevant role in limiting the ultimate beam emittance, as discussed in Sec. III D.

We note also that the photon energy of the ionization laser is not large enough to ionize directly ground-state Cs 
atoms. Other ionization processes involving more than one photon at 852 or $473 \mathrm{~nm}$ are possible, but their contribution is expected to be negligible in the actual conditions of the experiment due to the much smaller cross sections for multiphoton processes.

The use of photoionization enables completely disentangling the charge production and extraction steps. Therefore, ions can be extracted from the interaction volume by an externally applied electric field with an adjustable intensity. Such an electric field is produced by two parallel circular-shaped grids consisting of stainlesssteel wire grids with a diameter of $27 \mathrm{~mm}$ [G1 and G2; see Fig. 1(a)] positioned along the atom beam path and mutually spaced by $22 \mathrm{~mm}$. The diameter of the wires is $0.2 \mathrm{~mm}$ and the grid pitch is $1.8 \mathrm{~mm}$; the resulting geometrical transmission efficiency is approximately $77 \%$.

In a first approximation, G1 and G2 form a plane-parallel capacitor which acts as an ion accelerator and extractor. Grid G1 is connected to an adjustable positive voltage $V_{1}$, whereas grid $\mathrm{G} 2$ is grounded. The interaction region from where ions are extracted is placed in between G1 and $\mathrm{G} 2$. The produced ions having mass $m$ and charge $e$ are accelerated to a longitudinal velocity $v=\sqrt{v_{0}^{2}+\chi e V_{1} / m}$, where $v_{0}=v_{z} \approx 23 \mathrm{~m} / \mathrm{s}$ is the initial velocity of the produced ions assumed equal to the average longitudinal velocity of the slow atoms in the actual conditions of the present experiment, and $\chi=0.5-0.8$ is a factor depending on the actual ionization position. The resulting velocity is dominated significantly by the second term already for $V_{1}>1 \mathrm{mV}$. This confirms that the initial energy of the atoms does not play a significant role in ruling the energy of the extracted ions, at least for the $V_{1}$ range explored in the present experiment.

After crossing grid G2, ions enter a region $14.5 \mathrm{~cm}$ long delimited at the end by the third grid G3 and finally are collected by the ion detector, whose aperture (geometrical area $17 \times 17 \mathrm{~mm}^{2}$ ) is placed close to G3 (relative distance $\lesssim 1 \mathrm{~cm}$ ). The detector is a windowless electron multiplier (226 EM Thorn Emi) with its first dynode consisting of a curved $\mathrm{CuBe}$ plate biased at $-3000 \mathrm{~V}$ that provides a nominal gain approximately $5 \times 10^{7}$. The detector is operated in the counting mode, ion counts being measured by a gated digital counter (Stanford Research Systems SR400). The counter discriminator threshold is adjusted in order to minimize counts stemming from electromagnetic noise. Uncertainty of the measured count rate and ion counts is determined as the experimental standard deviation over a sample of repeated measurements.

\section{RESULTS AND DISCUSSION}

\section{A. Behavior of the collection and detection system}

The simple arrangement of G1, G2, G3 electrodes is responsible for extracting the ions and guiding them onto the detector. Diagnostics of the experiment is, hence, based on the ion collection and detection system, whose behavior is thoroughly investigated as a function of the bias voltages $V_{1}$ and $V_{3}$. Figure 2 shows the ion count rate as a function of $V_{1}$ for two distinct values of the $\mathrm{G} 3$ bias, i.e., $V_{3}=$ $-105 \mathrm{~V}$ and $V_{3}=0$ (filled and open circles, respectively). We find that the ion count rate after an initial increase follows a trend dominated by the dependence of the detector sensitivity on the ion-beam position and width. For $V_{1} \gtrsim 800 \mathrm{~V}$, the count rate saturates to a common value, roughly independent of $V_{3}$ in the explored range. At intermediate $\mathrm{G} 1$ bias, i.e., for $200 \mathrm{~V} \lesssim V_{1} \lesssim 800 \mathrm{~V}$, a nonmonotonic trend is observed, with a peak in the count rate.

In order to interpret the trend shown in Fig. 2, the electric field generated by the electrode arrangement has been simulated with the Poisson SuperFish code [42], assuming a cylindrical symmetry [the axis being along the $z$ direction; see Fig. 1(a)] for the electric field distribution. Ion trajectories in the actual experimental conditions are numerically reconstructed by the GPT software [43]. The results indicate that the average radius of the ion beam at the position of G3 and of the ion detector is an increasing function of $V_{1}$, with a pronounced slope for $V_{1}<200 \mathrm{~V}$. The average radius is also a slightly increasing function of $V_{3}$. For instance, the simulation leads to an average radius of 4.6 and $4.2 \mathrm{~mm}$ on $\mathrm{G} 3$ and on the ion detector planes, respectively, for $V_{3}=0$ and $V_{1}=100 \mathrm{~V}$.

The charge detector installed in our setup featuring a curved first dynode is known to exhibit a nonhomogeneous sensitivity as a function of the hitting position of the ions onto the first dynode (see, e.g., Ref. [44]). The circumstance combined with the dependence of the ion-beam size on $V_{1}$ and $V_{3}$, is mostly responsible for the observed behavior. By inferring the spatial dependence of the

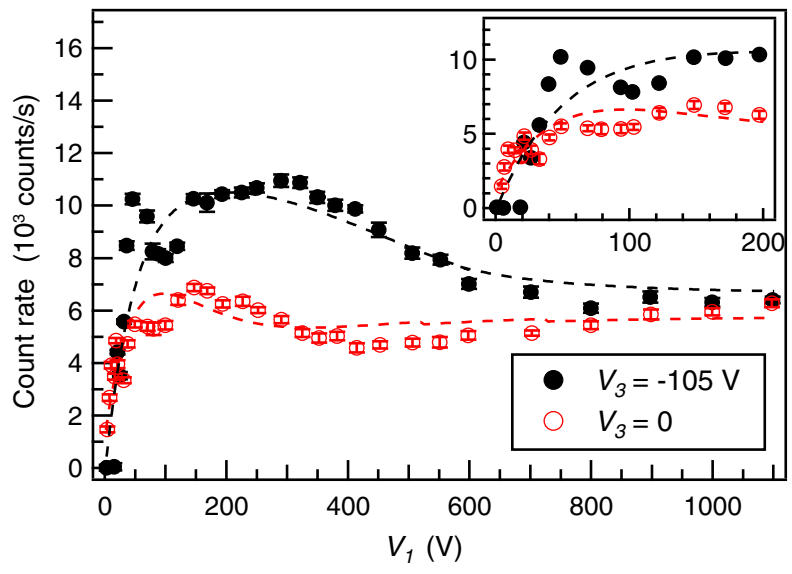

FIG. 2. Ion count rate as a function of $V_{1}$ for two distinct values of $V_{3}$, as specified in the legend. Dashed lines represent the expected behavior accounting for the detector response, as discussed in the text. The inset reports data at low $V_{1}$. Excitation laser power $P_{\mathrm{exc}}=1 \mathrm{~mW}$ and ionization laser power $P_{\text {ion }}=10 \mathrm{~mW}$. 
detector sensitivity from Ref. [44] and using the simulated average radius at the detector entrance, the trends shown as dashed lines in Fig. 2 are found. Furthermore, small oscillations are observed in the count rate, especially for $V_{1}<200 \mathrm{~V}$ and $V_{3}=-105 \mathrm{~V}$ (see the inset of Fig. 2), which can be ascribed to the transmission efficiency of the grids G2 and G3 used as electrodes. The ion yield can, in fact, decrease when the beam radius approaches a multiple of the metal grid pitch, i.e., when charge absorption by the metal wires takes place.

\section{B. Continuous- and pulsed-mode operation of the ion beam}

In the atom-light interaction process of Fig. 1(b), the ionization rate is $r_{\lambda}=\sigma_{\lambda} I_{\text {ion }} \lambda /(h c)$, with $I_{\text {ion }}$ the intensity of the ionization laser, $h, c$ the Planck's constant and light speed, respectively, and $\sigma_{\lambda} \approx 1.4 \times 10^{-17} \mathrm{~cm}^{2}[45,46]$ the ionization cross section at $\lambda=473 \mathrm{~nm}$. Therefore, the count rate is linearly dependent on the ionization laser power $P_{\text {ion }}$, as demonstrated by Fig. 3(a).
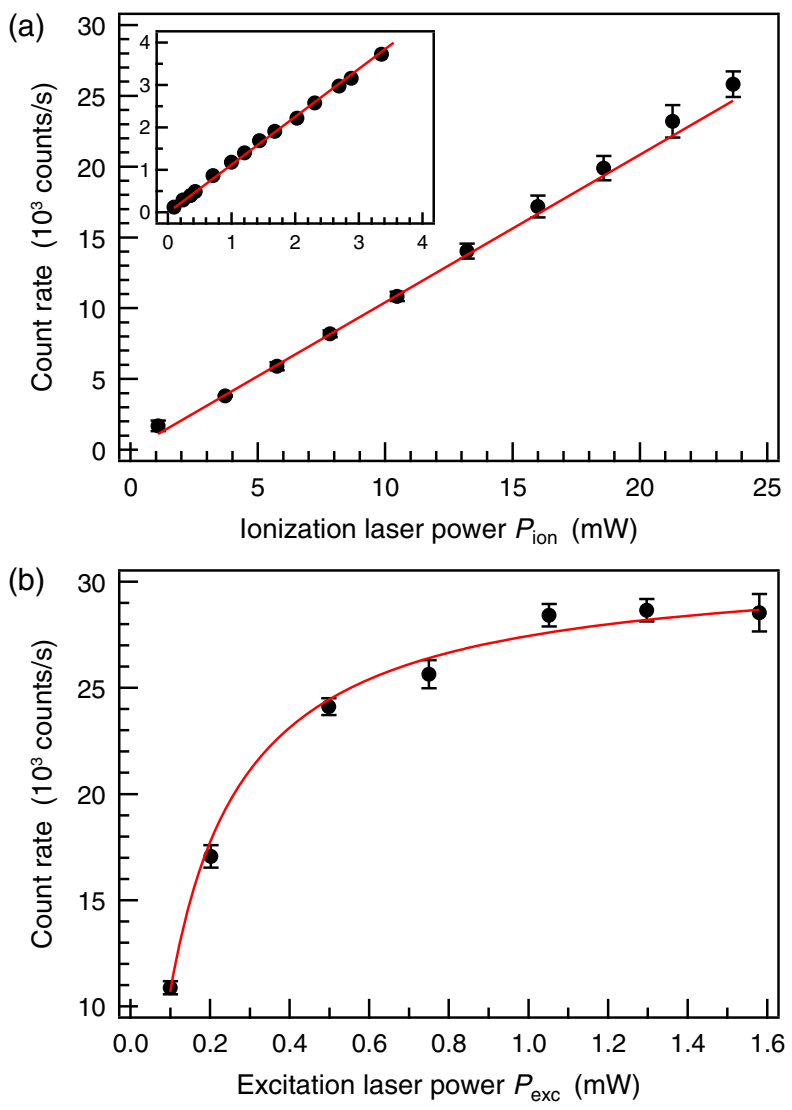

FIG. 3. Ion count rate versus ionization laser power $P_{\text {ion }}$ for $P_{\text {exc }}=1 \mathrm{~mW}$ (a) and versus excitation laser power $P_{\text {exc }}$ for $P_{\text {ion }}=25 \mathrm{~mW}$ (b); the solid lines represent a linear best fit (a) and the result of the model briefly outlined in Appendix A (b). The inset in panel (a) reports measurements at low ionization power. For all shown measurements, $V_{1}=300 \mathrm{~V}$ and $V_{3}=-105 \mathrm{~V}$.
The comparison between the slope of a linear best fit to the experimental data $A=(1.08 \pm 0.08) \times 10^{3}$ counts/ (s mW) and the expected value $A_{\text {est }}$ allows us to infer a collection and detection efficiency $\eta=A / A_{\text {est }} \sim 1 / 3$ (at $V_{1}=300 \mathrm{~V}$ and $V_{3}=-105 \mathrm{~V}$ ) limited by losses due, e.g., to ion trajectories falling outside the effective aperture size of the detector, to the absorption of charge by the grid wires, and to the quantum efficiency of the detector at the used operating voltage [44]. Since the available power for the ionization laser in the present experiment is $P_{\text {ion }} \lesssim 25 \mathrm{~mW}$, a maximum ion current $J \sim 10 \mathrm{fA}$ can be attained that makes cumbersome the absolute calibration of the system through comparison with an independent current measurement, e.g., by Faraday cup methods.

The behavior of the count rate as a function of the excitation laser power $P_{\text {exc }}$ shown in Fig. 3(b) reflects the occurrence of saturation for the selected atomic transition. The solid line superposed to the experimental data represents the result of the model briefly outlined in Appendix A.

The robustness of the pyramidal MOT able to produce a stable and reliable atom beam with moderate particle density and well-defined dynamical features [36] makes it an ideal starting source for the straightforward production of ion bunches delivering a predefined amount of charge. Two different techniques are explored to this aim: (i) the production of cesium atom bunches and subsequent ionization with $\mathrm{cw}$ lasers and (ii) pulsed excitation of the continuous cesium beam in the presence of a cw ionization laser.

In the first method, bunches of atoms are achieved by deflecting, i.e., pushing away, the atoms by optical pressure [39]. A laser beam resonant with the $6{ }^{2} S_{1 / 2}(F=4) \rightarrow 6$ ${ }^{2} P_{3 / 2}\left(F^{\prime}=5\right) \mathrm{Cs} \mathrm{HF}$ transition and delivering an intensity above saturation is sent orthogonally to the atom beam right after the 2D optical molasses region [see Fig. 1(a)], along with a weaker repumping beam tuned on the $6{ }^{2} S_{1 / 2}(F=3) \rightarrow$ $6{ }^{2} P_{3 / 2}\left(F^{\prime}=4\right) \mathrm{HF}$ transition. This radiation is needed to recycle atoms, enhancing the effectiveness of the process. A variable-length atom bunch is created by switching the pushing laser beam off for a predefined duration $\tau_{\text {push }}$. In other words, if the pushing laser beam is turned on, the atom beam is deflected out of the ionization region, and no ion is created, and vice versa. This technique involving blanking the atom beam allows us to virtually rule out any neutral atom background in the interaction volume.

The number of ionized atoms is proportional to the bunch length, $v_{0} \tau_{\text {push }}$. The delay between switching off the pushing laser beam and ionization of the atoms subsequent to their arrival into the interaction region is used in a time-of-flight approach to determine the average velocity of the atom beam, resulting in $v_{0}=(22.6 \pm 0.1) \mathrm{m} / \mathrm{s}$ in the actual conditions of the experiment, as well as the width of its Maxwellian velocity distribution, $\Delta v=(3.20 \pm 0.04) \mathrm{m} / \mathrm{s}$. 

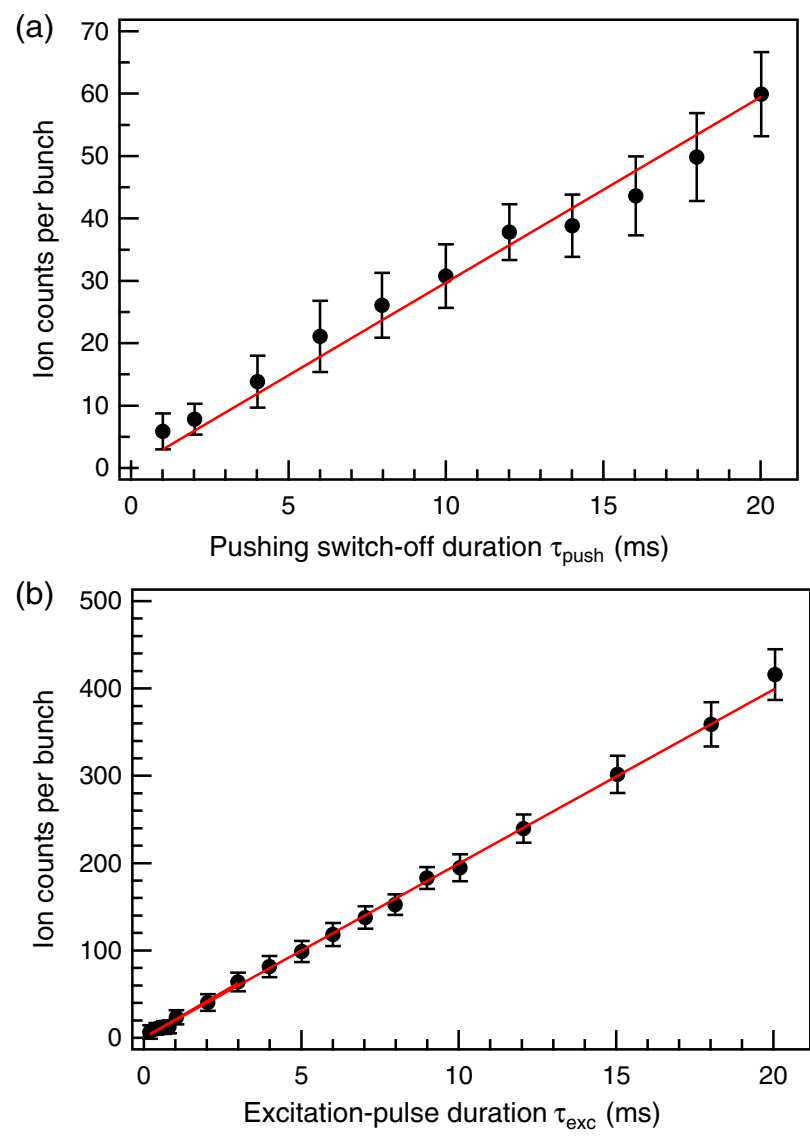

FIG. 4. Ion counts as a function of laser-pulse duration for ion bunches produced through (a) the pushing method (see text) and (b) the pulsed-excitation method. The solid lines represent linear fits to the experimental data. For all measurements, $V_{1}=300 \mathrm{~V}$ and $V_{3}=-105 \mathrm{~V}, P_{\text {exc }}=2 \mathrm{~mW}, P_{\text {ion }}=20 \mathrm{~mW}$.

Data shown in Fig. 4(a) demonstrate the expected linear behavior as a function of $\tau_{\text {push }}$, at least in the explored range. They are well described by a straight line with slope $R=(3.0 \pm 0.2)$ ions $/ \mathrm{ms}$, as derived from a best-fit procedure. However, deflecting the beam out and in the interaction region takes a non-negligible time mainly due to the inertia of the atoms changing their direction of motion. This circumstance prevents the controlled operation of the method at arbitrarily short pulse duration and sets limitations in the ability to shape the single bunch. As a consequence, data acquired with sub-ms $\tau_{\text {push }}$, not shown here, tend to deviate from linearity, making the approach unsuitable to achieve the desired charge-number control in very small ion bunches.

In the second ion-bunch production method, we make the continuous laser-cooled atom beam interact with a pulsed-excitation laser in the presence of $\mathrm{cw}$ ionization radiation. Excitation laser pulses of a predefined duration $\tau_{\text {exc }}$ are produced by means of an electro-optic modulator (EOM, Gsaenger LM 0202). The results are shown in Fig. 4(b) as a function of $\tau_{\text {exc }}$. Also in this case, a linear trend is observed, in agreement with the expectations, with a slope $R^{\prime}=(20 \pm 3)$ ions $/ \mathrm{ms}$. The larger slope value compared to the $R$ for the beam-deflection method demonstrates a larger efficiency for the pulsed-excitation approach. This method is able to produce ion bunches with a duration limited only by the bandwidth of the EOM and of its control electronics (in the magahertz for our setup). Moreover, by carefully shaping the laser pulse in order to have practically null power in off conditions, the background counts are negligible.

\section{Energy and energy-spread analysis}

The average energy and relative energy spread of an ion beam are, in general, relevant figures of merit to assess its applicative potential. The measurement of such quantities is, hence, an essential step towards the characterization of the source.

In our setup, the ion-extraction energy can be tuned by acting on a well-controlled physical quantity not involved in the charge-creation process, i.e., the bias voltage $V_{1}$. In ion beams possessing a low kinetic energy, space-charge effects as well as the related disorder-induced heating can remarkably affect the energy distribution $[33,47]$, producing uncontrolled modifications in both longitudinal and transversal velocities. Such effects come into play when large particle densities occur and are, in general, particularly severe in the ion-creation stage, where they are often mitigated through the application of strong extracting fields. Owing to the relatively small ionization rate occurring in our experiment, space-charge and related effects can be assumed negligible. As a matter of fact, with the available ionization laser power, an ion production rate $\lesssim 10^{4}$ ions/s is attained, which means an average time of hundreds of microseconds is spent within subsequent ionization events. In such a relatively long time interval, ions are displaced from each other by a distance expectedly so large to rule out mutual Coulomb interaction effects.

Ruling out such effects allows us to use a simple model neglecting space charge [24], where the rms energy spread is $\Delta U_{\mathrm{rms}} \propto \sigma_{\mathrm{rms}} E_{0}$, with $\sigma_{\mathrm{rms}}$ the rms width $(1 / \sqrt{e}$ intensity radius) of the ionizing beam, smaller than the excitation beam in our setup, and $E_{0}$ the amplitude of the electric field in the interaction region assumed homogeneous. The ion average energy and energy spread can be measured by different methods including the retarding field-energyanalyzer (RFEA) diagnostics [48], which has been used in many contexts [49-51]. We implement the RFEA method by duly biasing the electrode configuration installed in our setup. In particular, we adjust the bias voltage applied to grid G1 in order to modify $E_{0}$, hence, the average energy $U$ of the extracted ion beam. The retarding or stopping potential $V_{\text {stop }}$ is provided by applying a positive voltage to G3. A RFEA measures the integral over that part of the distribution function above the stopping energy $U_{\text {stop }}=e V_{\text {stop. Assuming a Gaussian }}$ 
energy-distribution function for the extracted ions, a planar grid potential with equipotential surfaces normal to the velocity of the incoming particles, neglecting grid thickness and the dependence of the detection sensitivity on the energy of the ions, the count rate $N_{i, \text { det }}$ is given by

$$
N_{i, \mathrm{det}}=C[1+\operatorname{erf}(\beta)],
$$

with erf the error function, $C$ a normalization factor, and

$$
\beta=\frac{U-U_{\text {stop }}}{\sqrt{2} \Delta U_{\text {rms }}} .
$$

The detected count rate $N_{i, \text { det }}$ as a function of the stopping potential $V_{\text {stop }}$ can be, hence, used for evaluating $\Delta U_{\text {rms }}$ and $U$ through a best fit of data to Eq. (1), leaving $C$ as an additional fitting parameter. Figure 5 summarizes the results for $\Delta U_{\text {rms }}$ as a function of $V_{1}$ in the range $10-80 \mathrm{~V}$. The plot reports data obtained with both copropagatingand crossed-laser-beam configurations (open and filled circles, respectively). The reported uncertainties are provided by the best-fit procedure to Eq. (1), being the error bars in $N_{i, \text { det }}$ given by the experimental standard deviation

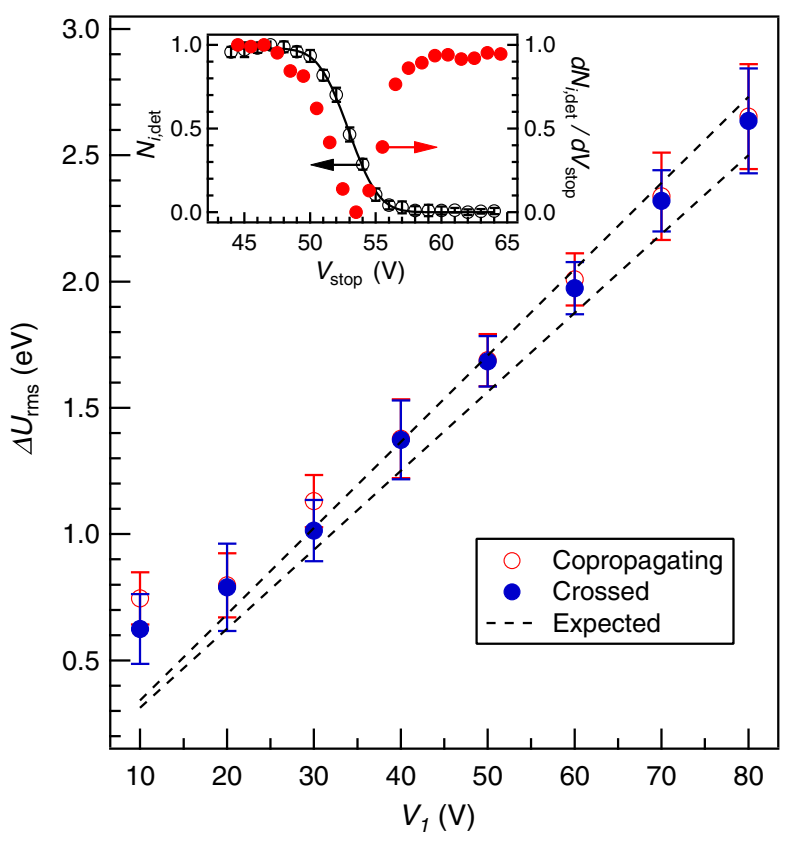

FIG. 5. Ion-beam rms energy spread $\Delta U_{\text {rms }}$ as a function of the bias voltage $V_{1}$, in the copropagating- and crossed-laser-beam configurations (open and filled circles, respectively). The expected values calculated using $E_{0}$ determined through numerical simulations of the electric field are shown with dashed lines (two lines are used to account for experimental uncertainties in the relevant quantities entering the equations). The inset shows an example of $N_{i, \text { det }}$ and $d N_{i, \text { det }} / d V_{\text {stop }}$, both normalized to unity, as a function of $V_{\text {stop }}$ (open and filled circles, respectively). The superposed solid line represents the result of the best fit to Eq. (1). For all measurements $P_{\text {exc }}=2 \mathrm{~mW}$ and $P_{\text {ion }}=25 \mathrm{~mW}$. over a sample of repeated measurements. The expected trend calculated by using the amplitude $E_{0}$ obtained by numerical simulations of the electric field in the actual bias conditions of the experiment is also shown with dashed lines.

The inset of Fig. 5 reports an example of the count rate as a function of $V_{\text {stop }}$; for the sake of clarity, the count rate is normalized to unity (i.e., the normalization factor is fixed to $C=1)$. Data represented by open circles are well reproduced by the function of Eq. (1) and reported as a solid line. The result of a numerical differentiation $d N_{i, \text { det }} / d V_{\text {stop }}$ is also shown (filled circles), leading to a Gaussian-like function. The parameters obtained by the best-fit procedure for this specific example are $U=(52.9 \pm 0.3) \mathrm{eV}$ and $\Delta U_{\mathrm{rms}}=(1.7 \pm 0.1) \mathrm{eV}$; the relative rms energy spread is, therefore, $\Delta U_{\mathrm{rms}} / U=0.032 \pm 0.002$.

The measured energy spread increases with the ion-beam energy ruled by $V_{1}$ and agrees with the expectations for $V_{1} \geq 30 \mathrm{~V}$. For $V_{1}<30 \mathrm{~V}$, a rms energy spread below $1 \mathrm{eV}$ is found. We note that the data point acquired at the lowest G1 bias $V_{1}=10 \mathrm{~V}$ deviates from the expectations for a quantity larger than the error bar. Since the error bar is related to the experimental standard deviation of $N_{i, \text { det }}$ data, it does not include any possible systematic error associated with the use of RFEA at low energy. The discrepancy between the data and expectation can be related to several mechanisms [52-54] such as the nonplanar electric field, owing to the grid structure and thickness leading to transverse-electric field components, the residual dependence of the collection and detection efficiency on the ion energy, and the inhomogeneities of the electric field lines in the interaction volume. The role played by the latter effect can be evidenced by the use of the crossed configuration for the excitation and ionization laser beams (filled circles in Fig. 5). In such conditions, a smaller transverse size [along the $x$ direction; see Fig. 1(a)] of the interaction volume is achieved, defined by the width of the excitation laser beam $\left(\sigma_{\text {rms,exc }} \approx 0.7 \mathrm{~mm}\right)$ rather than by the transverse size of the atom beam $\left(\sigma_{\text {rms,beam }} \approx 3.4 \mathrm{~mm}\right)$. As a consequence, ions are produced close to the system axis, where a more homogeneous electric field is expected. This circumstance slightly reduces the discrepancy between data and expectation also for $V_{1}=10 \mathrm{~V}$.

\section{Emittance, chromaticity, and brightness of the ion source}

An estimation of the figures of merit typical for conventional FIBs, i.e., emittance and brightness [1,28], is useful to evaluate the capabilities of our approach in the present configuration or assuming simple and straightforward experimental implementations.

The emittance $\epsilon^{\prime}$ is a measure of the phase space occupied by the beam particles. At a beam focus, e.g., in the region where the beam is created, it can be expressed (in one direction) as the product of the ion-beam width $\sigma_{i}$ 
with its angular spread $\alpha_{i}$. The photoionization process provides to the ions a velocity $v_{\mathrm{exc}} \simeq 1 \mathrm{~m} / \mathrm{s}$ along all directions, including the transversal one (see Sec. II), whereas the longitudinal velocity is dominated by that of the atom beam $\left(v_{0} \approx 23 \mathrm{~m} / \mathrm{s}\right)$. We can, therefore, estimate $\alpha_{i}=v_{\text {exc }} / v_{0} \sim 40 \mathrm{mrad}$ in the conditions of the present experiment. This value will obviously decrease by using ionization laser wavelengths close to the threshold value around $508 \mathrm{~nm}$ [41], as enabled, for instance, by commercial solid-state lasers at $501 \mathrm{~nm}$ or by the recently demonstrated source at $509 \mathrm{~nm}$ [55], leading to $\alpha_{i}<10 \mathrm{mrad}$. We cannot rule out using inexpensive diode lasers at $510 \mathrm{~nm}$, mounted in the external cavity configuration in order to get the required tunability and attain an even smaller initial divergence. In any case, the divergence of the atom beam sets a lower bound for $\alpha_{i}$. Even in the case of near-threshold ionization, $\alpha_{i} \simeq \alpha \approx 4 \mathrm{mrad}$.

In the copropagating configuration used to enhance the signal-to-noise ratio during the measurements, $\epsilon^{\prime} \simeq$ $140 \mathrm{~mm}$ mrad is estimated, being $\sigma_{i}=\sigma_{\text {rms,beam }} \approx 3.4 \mathrm{~mm}$. In the crossed-beam configuration, the transverse size of the ion production region is ruled by that of the laser beams, leading to a smaller emittance. In order to assess the potential of our approach, we can suppose to focus our laser beams to a waist $\left(1 / e^{2}\right.$ intensity radius) of, for instance, $w \simeq 5 \mu \mathrm{m}$, also feasible from the experimental point of view [56].

Neglecting space-charge and aberration effects, the energy-normalized emittance defined as $\epsilon_{x}=\epsilon_{x}^{\prime} \sqrt{U}$ is an invariant quantity along the ion-focusing path $[1,28]$. Therefore, the smaller the normalized emittance, the higher the ability to tightly focus the beam. In our experimental conditions, where the temperature $T$ plays a dominant role in determining the transverse motion of the ions, the normalized emittance (rms) can be conveniently expressed as $\epsilon_{x}=\sigma_{i} \sqrt{k_{B} T / 2}[22,28]$, with $k_{B}$ the Boltzmann's constant. For ionization at $473 \mathrm{~nm}$, where an excess energy $U_{\text {exc }}$ is provided to the ions by photoionization, $k_{B} T \sim U_{\text {exc }}$. In the case of photoionization at threshold, we can assume $k_{B} T \sim k_{B} T_{\perp}$, with $T_{\perp}$ the transverse temperature of the laser-cooled atoms, on the order of the Doppler temperature for cesium $\left(T_{D} \simeq 125 \mu \mathrm{K}\right.$ [41]).

Assuming laser-beam waists $w \simeq 5 \mu \mathrm{m}$ corresponding to a rms width $\sigma=w / 2=2.5 \mu \mathrm{m}$, the calculated normalized emittance amounts to $\epsilon_{x} \sim 2 \times 10^{-6} \mathrm{mrad} \mathrm{mm} \sqrt{\mathrm{MeV}}$ for photoionization at $473 \mathrm{~nm}$ and $\epsilon_{x} \sim 1 \times$ $10^{-7} \mathrm{mradmm} \sqrt{\mathrm{MeV}}$ for at threshold photoionization. The latter value, while being larger than the expected performance of recently proposed approaches based on laser cooling of effusive beams [25,32], is smaller than for the liquid-metal tip sources widely diffused in commercial FIBs, as well as for some sources based on the ionization of laser-trapped atoms $[22,24,57]$. Furthermore, thanks to the distinctive feature of our setup consisting in the ionization of a low-particle-density beam, we can safely disregard any unwanted increase of the emittance due to space-charge and related effects.

The beam chromaticity, i.e., the energy spread of the ions extracted from the ionization volume, is also a relevant parameter to determine the ultimate focusing abilities of the apparatus. In the range of extraction voltages explored, the energy spread is roughly linearly dependent on the extraction energy. Thanks to the absence of space-charge and related effects, such a linear behavior is expected to hold also at very low extraction energy, with a lower bound imposed by the initial energy of the ions $U_{\text {exc }}$. By supposing laser-beam waists $w \simeq 5 \mu \mathrm{m}$, a rms relative energy spread $\Delta U_{\text {rms }} / U \sim 2 \times 10^{-4}$ is expected, independent of the energy $U$. The performance is similar to that of state-of-the-art liquid-metal tip sources reported to have typical $\Delta U \approx 5 \mathrm{eV}$ at $U \sim 30 \mathrm{keV}$ [58]. A higher degree of monochromaticity is foreseen in some proposals of ion beams based on laser cooling [24,26], even though, owing to the large available current, in those sources space-charge and related effects can play a relevant role $[26,32,47]$ in degrading the chromatic properties.

Following the approach presented in Ref. [33], the relevance of aberrations in focusing our beam can be estimated assuming that ions are coupled to a conventional FIB column. As detailed in Appendix B, the extrapolated minimum spot size for an acceleration voltage of $30 \mathrm{kV}$ is slightly below $10 \mathrm{~nm}$, dominated by the chromatic aberrations. A further decrease of the energy spread can be achieved either by more tightly focusing the ionization laser beam or by reducing the electric field strength $E_{0}$ in the extraction region. Since in the plane-parallel-capacitor approximation $E_{0} \simeq V_{1} / d$, this reduction can be realized by simply increasing the distance $d$ between grids G1 and G2.

Finally, although our setup is not conceived by design to operate at large ion currents, evaluation of the brightness can be of interest in order to better point out the pitfalls of our approach. The brightness is a measure of the ability to convey current into the focal spot. In particular, the peak normalized brightness can be evaluated as $B_{\text {peak }}=$ $J /\left(4 \pi^{2} \epsilon_{x} \epsilon_{y}\right)$ [28], with $J$ the ion current and $\epsilon_{x, y}$ the normalized emittance along the transverse directions (for the sake of simplicity, we will assume here $\epsilon_{x}=\epsilon_{y}$ ). In the copropagating configuration, a maximum current $J \sim 10 \mathrm{fA}$ is obtained, which will drop below $0.1 \mathrm{fA}$ for the crossed configuration with focused laser beams. The corresponding peak-normalized brightness will be of a few hundreds $\mathrm{A} /\left(\mathrm{m}^{2} \mathrm{sreV}\right)$ in the best conditions of photoionization at threshold, a value smaller than for both stateof-the-art FIB machines and proposed or demonstrated sources based on laser-cooling [25,26,32,33]. The small available current in our setup is essentially due to the small atom flux achievable out of the pyramidal MOT. This limitation can, in principle, be circumvented by 
implementing complicated modifications, which will affect the overall simplicity of the approach and, consequently, the possibilities of a seamless integration in practical instrumentation. To this aim, the use of a larger pyramid, with the associated need for higher power in the laser trapping and cooling beams [40], and the implementation of a compression stage after the 2D optical molasses $[26,32]$ can effectively improve the atom flux.

\section{CONCLUSIONS}

We demonstrate the feasibility of a low-energy and lowcurrent ion source operated in continuous and pulsed modes. This source is based on two-color photoionization of the laser-cooled cesium beam produced out of a pyramidal MOT. Thus, it benefits from the large degree of control on the dynamical properties of neutral atoms enabled by laser trapping and cooling.

The original component of our implementation with respect to already proposed or demonstrated laser-cooling applications to ion sources (see, e.g., Refs. [20,22$27,32,33,57])$ is the pyramidal MOT. It ensures by design several technical advantages with respect to configurations reported so far, in particular, conventional 2D and 3D MOTs. Requiring a single trapping and cooling laser beam, it allows for a substantial simplification of the optical setup and the related need for alignment, contributing to the system stability and resilience. Moreover, it can be integrated in a straightforward way with the UHV components devoted to ion guiding and focusing. Finally, the simple ionization scheme implemented here, as well as the trapping and cooling process, can be accomplished by readily available solid-state lasers suitable for technological exploitations.

Our approach benefits from the reliable and stable operation of a well-collimated atom beam with a small flux, which can be even set below the $10^{8}$ atoms/s range by simply decreasing the current flowing through the dispensers [59]. Such a distinctive feature, which, to the best of our knowledge, is hard to achieve with conventional (e.g., effusive) beams, represents an added value for the straightforward production of a low-current ion beam with wellcontrolled properties and the ability to operate at low energy thanks to the absence of space-charge and related effects.

Our experiment demonstrates that the delivery of a controlled number of ions, ideally down to the singleion level, can be effectively attained by simply pulsing either a pushing or excitation laser beam. Concerning the deterministic nature of the process, our system is still statistical in nature. Nevertheless, given the very high collection efficiency that can be reached also for electrons, ion delivery can be cross-checked by electron detection, resulting in an almost deterministic delivery of single ions.

The relative energy spread of the ion beam is investigated by implementing a variant of the RFEA method. Measurements are carried out in the continuous mode, but the results are expected to hold also for the pulsed operation. A relative energy spread $\Delta U_{\text {rms }} / U \simeq 0.032$ is achieved. The data confirm that, thanks to the simple electrode configuration, the average energy can be adjusted by controlling the bias voltage of a single electrode. The energy spread depends on the size of the interaction volume. For applications requiring low ion current, where the production of sub-femtoampere current is not a concern, focusing of the laser beams down to the 5- $\mu \mathrm{m}$ waist is feasible, leading to an extrapolated relative energy spread $\Delta U_{\text {rms }} / U \sim 2 \times 10^{-4}$. The absence of space charge permits using simple calculations to determine the minimum achievable ion spot size. A spot diameter slightly below $10 \mathrm{~nm}$ is extrapolated assuming a crossed configuration for focused-excitation- and ionization-laser beams and photoionization at threshold.

Our approach is expected to be useful in those applications where the well-controlled production of low-current and mildly accelerated beams is required, as in a number of emerging applications of ion and surface interaction including, e.g., precise doping of thin layers [12-14], site-selective cellular implantation [11], and analytical and reactive surface processes [19].

\section{ACKNOWLEDGMENTS}

G. S., A.F., I. G., D. C., M. V., and F.F. gratefully acknowledge the support of the European Union Seventh Framework Program under Grant No. 251391 MC- IAPP "COLDBEAMS." The authors are deeply indebted to Daniel Comparat and Ennio Arimondo for extremely fruitful discussions and suggestions and to Nicola Puccini and Enrico Andreoni for their invaluable technical assistance in the experiment.

\section{APPENDIX A: SATURATION OF THE EXCITED TRANSITION}

In closed two-level atomic systems, saturation of the excited-state population $Y$ is described by

$$
Y=B \frac{1}{1+I_{\mathrm{sat}} / I_{\mathrm{exc}}},
$$

where $B$ is a normalization factor, $I_{\mathrm{exc}}$ is the excitation intensity, and $I_{\text {sat }}$ represents the saturation intensity. The conventional saturation intensity for the considered Cs transition is $1.1 \mathrm{~mW} / \mathrm{cm}^{2}$, whereas a more accurate value accounting for the linear polarization of the excitation beam is $2.1 \mathrm{~mW} / \mathrm{cm}^{2}$ [41].

The population of excited states in multilevel atoms interacting with laser light is governed by several phenomena. In the specific conditions of our experiment, enabling relatively long atom-laser interaction times due to the small longitudinal velocity of the atoms, optical pumping plays a prominent role. Its effects, thoroughly analyzed in Ref. [60], where a similar experimental setup (nonoptimized for ion 
extraction) is used, include transient modifications of the branching ratio $\Pi$ as the atoms cross the excitation laser beam, leading to a deviation from $\Pi=1$, holding in case of closed systems, to an effective value [61], $\Pi_{\text {eff }}<1$.

A simplified picture of semiopen two-level systems can be given assuming a leakage of population from the selectively excited $F^{\prime}$ state occurring because of laser coupling to other excited HF levels $\left(F^{\prime}=3,4\right)$. Atoms excited to $F^{\prime}=4$ decay to the $F=3$ in the ground state, opening a leak channel for the transition. In a rough model, where a small leak rate independent of $I_{\text {exc }}$ is considered [62], the following function is expected to describe the upper-state population at the steady state:

$$
Y \simeq B \frac{I_{\mathrm{exc}} / I_{\mathrm{sat}}-\Lambda / \Gamma}{1+I_{\mathrm{exc}} / I_{\mathrm{sat}}},
$$

where $\Gamma=5.2 \mathrm{MHz}$ is the natural linewidth of the transition [41] and $\Lambda$ represents the leak rate. A best fit of the experimental data according to Eq. (A2) shown as a solid line in Fig. 3(b) returns a leak rate $\Lambda \sim 10^{4} \mathrm{~Hz}$, i.e., much smaller than the optical pumping rate $\Gamma$. The obtained value of the saturation intensity $I_{\text {sat }}=(2.7 \pm 0.8) \mathrm{mW} / \mathrm{cm}^{2}$ is in agreement with the value reported in the literature [41]. We note that due to the small number of experimental points and to the relatively large error bars, we cannot reliably assess the validity of the simplified model presented here compared to the closed two-level system of Eq. (A1).

\section{APPENDIX B: ABERRATION EFFECTS}

The diameters $d_{C, 50}$ and $d_{S, 50}$ of the disks containing one-half of the ion current displaced from the spot because of chromatic and spherical aberration, respectively, are given by $[33,63]$

$$
\begin{gathered}
d_{C, 50}=0.811 C_{C} \frac{\Delta U_{\mathrm{rms}}}{U} \alpha_{L}, \\
d_{S, 50}=\frac{1}{4 \sqrt{2}} C_{S} \alpha_{L}^{3},
\end{gathered}
$$

where $C_{C}$ and $C_{S}$ are constructive parameters of the FIB column, and $\alpha_{L}$ is the angular aperture of the ion focusing lens. Assuming that the emittance contribution to aberration has a Gaussian distribution [28], $\alpha_{L}$ can be related to the diameter $d_{50}$ of the focal spot arising from beam emittance through

$$
\alpha_{L}=\sigma_{i} \frac{4 \sqrt{\ln 2}}{d_{50} v_{/ /}} \sqrt{\frac{k_{B} T}{m}}
$$

where $v_{/ /}=\sqrt{2 U_{\text {acc }} / m}$, with $U_{\text {acc }}$, the energy of the accelerated beam, is the longitudinal velocity of the ions in the focal spot. In order to highlight the role of the normalized beam emittance $\epsilon_{x}$, the latter equation can be conveniently rewritten as [28]

$$
\alpha_{L}=\epsilon_{x} \frac{4 \sqrt{\ln 2}}{d_{50} \sqrt{U_{\mathrm{acc}}}}
$$

Following Ref. [33], we can put $U_{\text {acc }}=30 \mathrm{keV}$, as is typical for FIBs, $C_{C}=0.1 \mathrm{~m}$, and $C_{S}=0.85 \mathrm{~m}$. The actual spot diameter $d_{T, 50}$ accounting for the contributions of chromatic and spherical aberration can then be estimated as $[28,33]$

$$
d_{T, 50}=\sqrt{\left(d_{50}^{1.3}+d_{S, 50}^{1.3}\right)^{\frac{2}{1.3}}+d_{C, 50}^{2}}
$$

Figure 6 shows $d_{C, 50}, d_{S, 50}$, and $d_{T, 50}$ (dotted, dashed, and solid line, respectively) as a function of $d_{50}$ calculated by putting $\Delta U_{\mathrm{rms}} / U=2 \times 10^{-4}$ in Eq. (B1) and $\sigma_{i}=$ $2.5 \mu \mathrm{m}$ in Eq. (B3) [that is, $\epsilon_{x}=10^{-7} \mathrm{~mm} \mathrm{mrad} \sqrt{\mathrm{MeV}}$ in Eq. (B4)]. Chromatic aberration dominates over spherical aberration in all the explored $d_{50}$ range. Because of the specific dependence on $\alpha_{L}$ of the different quantities entering Eq. (B5), the calculated actual spot diameter shows a minimum for $d_{T, 50}$ slightly below $10 \mathrm{~nm}$, which can be considered as the extrapolated minimum spot size achievable with our ion source. Owing to the dominant role of chromatic aberration, the focusing abilities are mostly limited by the residual relative energy spread of the source, which can be improved either by more tightly focusing the ionization laser beam or by reducing the electric field strength $E_{0}$ in the extraction region.

We remark, however, that the calculation presented in this appendix should be regarded as a very rough estimation being based on several strong and not verified assumptions on the behavior of the ion-guiding and -focusing stage, as duly outlined in Ref. [28].

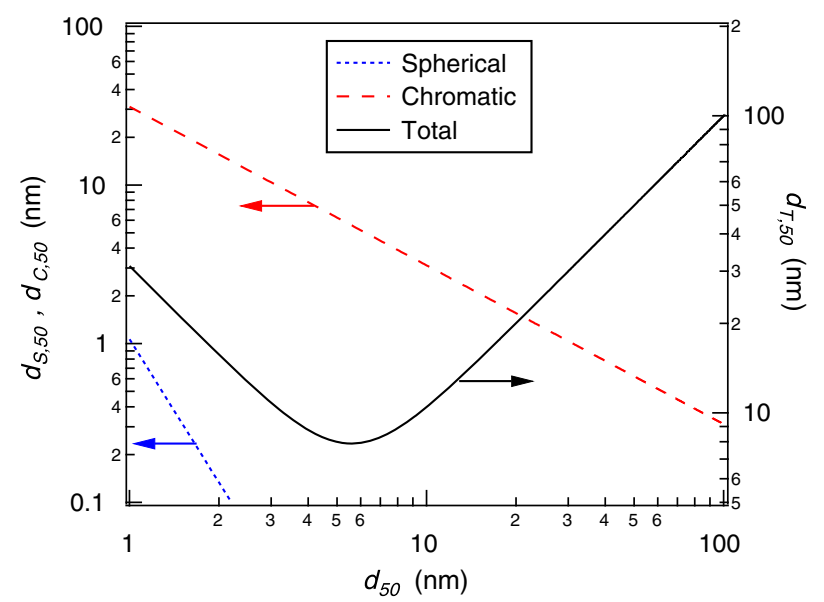

FIG. 6. Calculated diameter of the disks produced by the contribution of spherical and chromatic aberration $d_{S, 50}$ and $d_{C, 50}$, respectively, as a function of the diameter $d_{50}$ of the focal spot arising from beam emittance. The actual spot diameter $d_{T, 50}$ accounting for aberration is also reported. 
[1] J. Orloff, M. Utlaut, and L. Swanson, High Resolution Focused Ion Beams: FIB and Its Applications (SpringerVerlag, New York, 2002).

[2] See, for instance, http://www.fei.com; http:// www.orsayphysics.com; http://www.zeiss.com/microscopy.

[3] N. Bassim, K. Scott, and L. A. Giannuzzi, Recent advances in focused ion beam technology and applications, MRS Bull. 39, 317 (2014).

[4] I. Ohdomari, Single-ion irradiation: Physics, technology and applications, J. Phys. D 41, 043001 (2008).

[5] P. M. Koenraad and M. E. Flattè, Single dopants in semiconductors, Nat. Mater. 10, 91 (2011).

[6] J. C. McCallum, D. N. Jamieson, C. Yang, A. D. Alves, B. C. Johnson, T. Hopf, S. C. Thompson, and J. A. van Donkelaar, Single-ion implantation for the development of Si-based MOSFET devices with quantum functionalities, Adv. Mater. Sci. Eng. 2012, 272694 (2012).

[7] A. Persaud, S. J. Park, J. A. Liddle, T. Schenkel, J. Bokor, and I. W. Rangelow, Integration of scanning probes and ion beams, Nano Lett. 5, 1087 (2005).

[8] J. Meijer, T. Vogel, B. Burchard, I. W. Rangelow, L. Bischoff, J. Wrachtrup, M. Domhan, F. Jelezko, W. Schnitzler, S. A. Schulz, K. Singer, and F. Schmidt-Kaler, Concept of deterministic single ion doping with sub-nm spatial resolution, Appl. Phys. A 83, 321 (2006).

[9] G. Jacob, K. Groot-Berning, S. Wolf, S. Ulm, L. Cuturier, S. T. Dawkins, U. G. Poschinger, F. Schmidt-Kaler, and K. Singer, Microscopy with a deterministic single ion-source, arXiv: $1512.00347 \mathrm{v} 1$.

[10] M. Folkard, B. Vojnovic, K. M. Prise, and B. D. Michael, The application of charged-particle microbeams in radiobiology, Nucl. Instrum. Methods Phys. Res., Sect. B 188, 49 (2002).

[11] T. Shinada, T. Akimoto, Y. Zhu, H. Goke, and I. Ohdomari, Modulation of viability of live cells by focused ion-beam exposure, Biotechnol. Bioeng. 108, 222 (2011).

[12] U. Bangert, A. Bleloch, M. H. Gass, A. Seepujak, and J. van den Berg, Doping of few-layered graphene and carbon nanotubes using ion implantantion, Phys. Rev. B 81, 245423 (2010).

[13] M. Telychko, P. Mutombo, M. Ondracek, P. Hapala, F. C. Bocquet, J. Kolorenc, M. Vondracek, and M. Svec, Achieving high-quality single-atom nitrogen doping of graphene/ $\mathrm{SiC}(0001)$ by ion implantation and subsequent thermal stabilization, ACS Nano 8, 7318 (2014).

[14] P. Wilke, J. A. Amani, A. Sinterhauf, S. Thakur, T. Kotzott, T. Druga, S. Weikert, K. Maiti, H. Hofsass, and M. Wenderoth, Doping of graphene by low-energy ion beam implantation: Structural, electronic, and transport properties, Nano Lett. 15, 5110 (2015).

[15] R. J. W. E. Lahaye and H. Kang, Reactive ion surface scattering as an Eley-Rideal process: A molecular dynamics study into the abstraction reaction mechanism by low energy $\mathrm{Cs}^{+}$from Pt(111), ChemPhysChem 5, 697 (2004).

[16] J. W. Rabalais, Principles and Applications of Ion Scattering Spectrometry: Surface Chemical and Structural Analysis (John Wiley and Sons, New York, 2002).

[17] J. B. Clegg, Optimum beam energy for high depth resolution secondary ion mass spectrometry, J. Vac. Sci. Technol. A 13, 143 (1995).
[18] A. Fiori, F. Jomard, T. Teraji, G. Chicot, and E. Bustarret, Improved depth resolution of secondary ion mass spectrometry profiles in diamond: A quantitative analysis of the delta doping, Thin Solid Films 557, 222 (2014).

[19] R. G. Cooks, T. Ast, T. Pradeep, and V. Wysocki, Reactions of ions with organic surfaces, Acc. Chem. Res. 27, 316 (1994).

[20] B. G. Freinkman, A. V. Eletskii, and S. I. Zaitsev, Laser ion beam formation for nanotechnologies, JETP Lett. 78, 255 (2003).

[21] B. J. Claessens, S. B. van der Geer, G. Taban, E. J. D. Vredenbregt, and O. J. Luiten, Ultracold Electron Source, Phys. Rev. Lett. 95, 164801 (2005).

[22] J. L. Hanssen, J. J. McClelland, E. A. Dakin, and M. Jacka, Laser-cooled atoms as a focused ion-beam source, Phys. Rev. A 74, 063416 (2006).

[23] J. L. Hanssen, S. B. Hill, J. Orloff, and J. J. McClelland, Magneto-optical-trap-based, high brightness ion source for use as a nanoscale probe, Nano Lett. 8, 2844 (2008).

[24] M. P. Reijnders, P. A. van Kruisbergen, G. Taban, S. B. van der Geer, P. H. A. Mutsaers, E. J. D. Vredenbregt, and O. J. Luiten, Low-Energy-Spread Ion Bunches from a Trapped Atomic Gas, Phys. Rev. Lett. 102, 034802 (2009).

[25] B. Knuffman, A. V. Steele, and J. J. McClelland, Cold atomic beam ion source for focused ion beam applications, J. Appl. Phys. 114, 044303 (2013).

[26] L. Kime, A. Fioretti, Y. Bruneau, N. Porfido, F. Fuso, M. Viteau, G. Khalili, N. Santic, A. Gloter, B. Rasser, P. Sudraud, P. Pillet, and D. Comparat, High-flux monochromatic ion and electron beams based on laser-cooled atoms, Phys. Rev. A 88, 033424 (2013).

[27] M. Viteau, M. Reveillard, L. Kime, B. Rasser, P. Sudraud, Y. Bruneau, G. Khalili, P. Pillet, D. Comparat, I. Guerri, A. Fioretti, D. Ciampini, M. Allegrini, and F. Fuso, Ion microscopy based on laser-cooled cesium atoms, Ultramicroscopy 164, 70 (2016).

[28] J. J. McClelland, A. V. Steele, B. Knuffman, K. A. Twedt, A. Schwarzkopf, and T. M. Wilson, Bright focused ion beam sources based on laser-cooled atoms, Appl. Phys. Rev. 3, 011302 (2016).

[29] F. Henkel, M. Krug, J. Hofmann, W. Rosenfeld, M. Weber, and H. Weinfurter, Highly Efficient State-Selective Submicrosecond Photoionization Detection of Single Atoms, Phys. Rev. Lett. 105, 253001 (2010).

[30] C. Ates, I. Lesanovsky, C. S. Adams, and K. J. Weatherill, Fast and Quasideterministic Single Ion Source from a Dipole-Blockaded Atomic Ensemble, Phys. Rev. Lett. 110, 213003 (2013).

[31] W. Schnitzler, N. M. Linke, R. Fickler, J. Meijer, F. Schmidt-Kaler, and K. Singer, Deterministic Ultracold Ion Source Targeting the Heisenberg Limit, Phys. Rev. Lett. 102, 070501 (2009).

[32] S. H. W. Wouters, G. ten Haaf, R. P. M. J. W. Notermans, N. Debernardi, P. H. A. Mutsaers, O. J. Luiten, and E. J. D. Vredenbregt, Performance predictions for a laser-intensified thermal beam for use in high-resolution focused-ion-beam instruments, Phys. Rev. A 90, 063817 (2014).

[33] G. ten Haaf, S. H. W. Wouters, S. B. van der Geer, E. J. D. Vredenbregt, and P. H. A. Mutsaers, Performance predictions of a focused ion beam from a laser cooled and 
compressed atomic beam, J. Appl. Phys. 116, 244301 (2014).

[34] K. A. Twedt, L. Chen, and J. J. McClelland, Scanning ion microscopy with low energy lithium ions, Ultramicroscopy 142, 24 (2014).

[35] C. O’Dwyer, G. Gay, B. Viaris de Lesegno, J. Weiner, A. Camposeo, F. Tantussi, F. Fuso, M. Allegrini, and E. Arimondo, Atomic nanolithography patterning of submicron features: Writing an organic self-assembled monolayer with cold, bright $\mathrm{Cs}$ atom beams, Nanotechnology 16, 1536 (2005).

[36] F. Tantussi, V. Mangasuli, N. Porfido, F. Prescimone, F. Fuso, E. Arimondo, and M. Allegrini, Towards lasermanipulated deposition for atom-scale technologies, Appl. Surf. Sci. 255, 9665 (2009).

[37] B. M. Xu, X. Chen, J. Wang, and M. S. Zhan, Realization of a single-beam mini magneto-optical trap: A candidate for compact CPT cold atom clocks, Opt. Commun. 281, 5819 (2008).

[38] S. Pollock, J. P. Cotter, A. Laliotis, F. Ramirez-Martinez, and E. A. Hinds, Characteristics of integrated magneto-optical traps for atom chips, New J. Phys. 13, 043029 (2011).

[39] A. Camposeo, A. Piombini, F. Cervelli, F. Tantussi, F. Fuso, and E. Arimondo, A cold cesium atomic beam produced out of a pyramidal funnel, Opt. Commun. 200, 231 (2001).

[40] J. J. Arlt, O. Maragò, S. Webster, S. Hopkins, and C. J. Foot, A pyramidal magneto-optical trap as a source of slow atoms, Opt. Commun. 157, 303 (1998).

[41] D. A. Steck, Cesium D line data, http://steck.us/alkalidata.

[42] J. H. Billen and L. M. Young, POISSON/SUPERFISH on PC Compatibles, in Proceedings of the 1993 Particle Accelerator Conference (IEEE, 1993), Vol. 2, pp. 790-792.

[43] See http://www.pulsar.nl/gpt.

[44] J. Krása, E. Woryna, and M. P. Stöckli, The effect of the first dynode's curvature on the gain of a type 226EM electron multiplier used as a detector of highly charged ions, Meas. Sci. Technol. 9, 1632 (1998).

[45] O. Maragò, D. Ciampini, F. Fuso, E. Arimondo, C. Gabbanini, and S. T. Manson, Photoionization cross sections for excited laser-cooled cesium atoms, Phys. Rev. A 57, R4110 (1998).

[46] B. M. Patterson, T. Takekoshi, and R. J. Knize, Measurement of the photoionization cross section of the $6 P_{3 / 2}$ state of cesium, Phys. Rev. A 59, 2508 (1999).

[47] A. V. Steele, B. Knuffman, and J. J. McClelland, Inter-ion coulomb interactions in a magneto-optical trap ion source, J. Appl. Phys. 109, 104308 (2011).

[48] J. A. Simpson, Design of retarding field energy analyzers, Rev. Sci. Instrum. 32, 1283 (1961).

[49] P. Y. Nabhiraj, R. Menon, G. Mohan Rao, S. Mohari, and R. K. Bhandari, Optimization of ion energy spread in inductively coupled plasma source designed for focused ion beam applications, Vacuum 85, 344 (2010).
[50] R. A. Rosenberg and K. C. Harkay, A rudimentary electron energy analyzer for accelerator diagnostics, Nucl. Instrum. Methods Phys. Res., Sect. A 453, 507 (2000).

[51] H. Ch. Paulini and U. Littmark, A simple and compact retarding field energy analyzer for the characterization of broad ion beams of low energy, Nucl. Instrum. Methods Phys. Res., Sect. B 58, 260 (1991).

[52] C. L. Enloe and J. R. Shell, Optimizing the energy resolution of planar retarding potential analyzers, Rev. Sci. Instrum. 63, 1788 (1992).

[53] Y. Cui, Y. Zou, A. Valfells, M. Reiser, M. Walter, I. Haber, R. A. Kishek, S. Bernal, and P. G. O'Shea, Design and operation of a retarding field energy analyzer with variable focusing for space-charge-dominated electron beams, Rev. Sci. Instrum. 75, 2736 (2004).

[54] P. D. Goldan, E. J. Yadlowsky, and E. C. Whipple, Jr., Errors in ion and electron temperature measurements due to grid plane potential nonuniformities in retarding potential analyzers, J. Geophys. Res. 78, 2907 (1973).

[55] M. Tawfieq, O. Bjarlin Jensen, A. Kragh Hansen, B. Sumpf, K. Paschke, and P. E. Andersen, Efficient generation of $509 \mathrm{~nm}$ light by sum-frequency mixing between two tapered diode lasers, Opt. Commun. 339, 137 (2015).

[56] We note that more tightly focused laser beams with a 3- $\mu \mathrm{m}$ waist are assumed, e.g., in Ref. [33], where the performances of an ion source based on photoionization of a laser-cooled effusive atom beam are comprehensively simulated.

[57] S. B. van der Geer, M. P. Reijnders, M. J. de Loos, E. J. D. Vredenbregt, P. H. A. Mutsaers, and O. J. Luiten, Simulated performance of an ultracold ion source, J. Appl. Phys. 102, 094312 (2007).

[58] C. W. Hagen and P. Kruit, Optimization of focused ion beam performance, J. Vac. Sci. Technol. B 27, 2654 (2009).

[59] N. Porfido, Ph.D. thesis, Università di Pisa, 2012.

[60] N. Porfido, N. N. Bezuglov, M. Bruvelis, G. Shayeganrad, S. Birindelli, F. Tantussi, I. Guerri, M. Viteau, A. Fioretti, D. Ciampini, M. Allegrini, D. Comparat, E. Arimondo, A. Ekers, and F. Fuso, Nonlinear effects in optical pumping of a cold and slow atomic beam, Phys. Rev. A 92, 043408 (2015).

[61] I. Sydoryk, N. N. Bezuglov, I. I. Beterov, K. Miculis, E. Saks, A. Janovs, P. Spels, and A. Ekers, Broadening and intensity redistribution in the $\mathrm{Na}(3 p)$ hyperfine excitation spectra due to optical pumping in the weak excitation limit, Phys. Rev. A 77, 042511 (2008).

[62] W. Demtröder, Laser Spectroscopy 2: Experimental Techniques, 5th ed. (Springer-Verlag, Berlin, 2015).

[63] Y. L. Wang and Z. Shao, Design principles of an optimized focused ion beam system, Adv. Electron. Electron Phys. 81, 177 (1991). 